\title{
Options for preserving fertility in women undergoing gonadotoxic treatment
}

\section{ABSTRACT}

Cancer chemotherapy and radiotherapy can be toxic to the ovaries, but women can improve their chances of preserving their fertility. Three options are available: gonadotropin-releasing hormone $(\mathrm{GnRH})$ analogues, oocyte cryopreservation, and ovarian tissue cryopreservation. A fourth option, ovarian transposition, is valid for patients undergoing pelvic radiation but is not useful in patients undergoing chemotherapy.

\section{KEY POINTS}

GnRH analogues provide only uncertain or temporary benefit, and should be offered only together with other options, or if other methods are not feasible.

Oocyte cryopreservation is now the standard of care and should be offered to all postpubertal patients who can wait at least 2 weeks before they start chemotherapy or radiotherapy.

Ovarian tissue cryopreservation is no longer experimental, although it poses a risk of reseeding in bloodborne cancers such as leukemia. It should be offered to prepubertal girls, who cannot undergo oocyte cryopreservation, and to postpubertal patients who do not have 2 weeks before starting therapy.
Medical Grand Rounds articles are based on edited transcripts from Medicine Grand Rounds presentations at Cleveland Clinic. They are approved by the author but are not peer-reviewed. doi:10.3949/ccjm.88gr.21001
ANy GIRLS AND YOUNG WOMEN with canL cer receive gonadotoxic chemotherapy or radiotherapy, which can threaten their ability to have children later on, or even put them into premature menopause (primary ovarian insufficiency). The probability of having a live birth is $30 \%$ to $50 \%$ lower in cancer survivors than in females without cancer. Even females who receive a moderate or low dose of chemotherapy and who do not experience primary ovarian insufficiency have significantly lower rates of conception., ${ }^{1,2}$

The risk of infertility is a source of considerable distress for women undergoing cancer treatment, as they typically have a very strong desire to have their own biological offspring after completing their treatment.,4 For these patients, proactive treatment can help preserve the possibility of having children.

\section{OVARIAN FOLLICLES MATURE IN STEPS}

The functional units of the ovary are the follicles in the ovarian cortex, each consisting of an egg (oocyte) surrounded by granulosa cells. These follicles mature in steps:

Primordial follicles are very small with only 1 layer of granulosa cells, which are flat or fusiform, surrounding the oocyte. Primordial follicles can progress to:

Primary follicles, in which the granulosa cells become more cube-shaped, and the egg is slightly bigger with a bigger cytoplasm. These in turn progress to:

Secondary follicles, in which the layers of granulosa cells increase. The cytoplasm around the nucleus of the egg increases in size, and so the egg increases in size as well.

Tertiary follicles, the last developmental stage, are characterized by the presence 


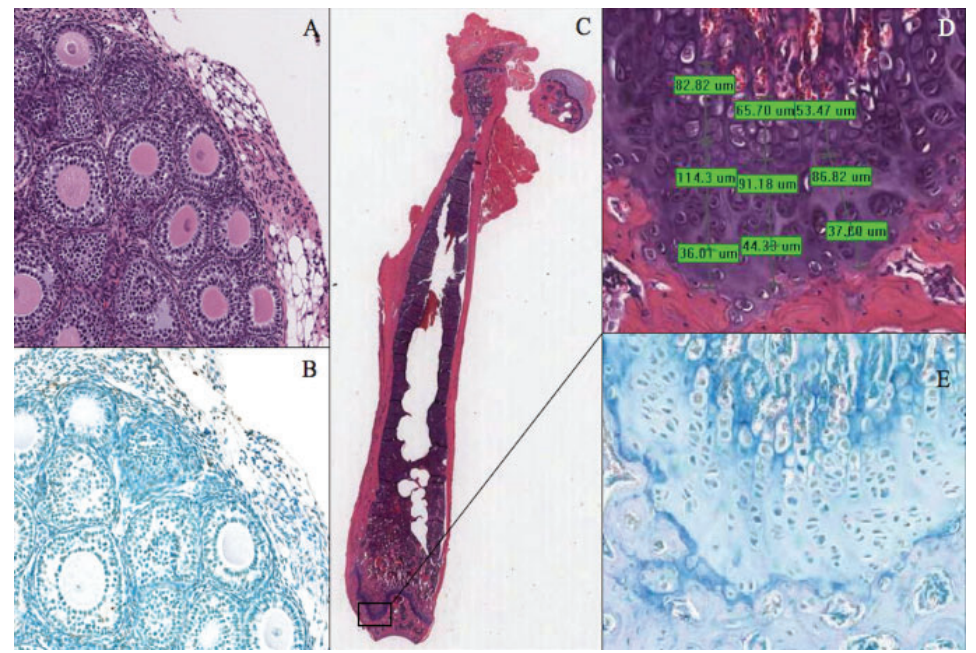

Figure 1. Ovarian follicles stained with hematoxylin and eosin (A) and with TUNEL (terminal nucleotidyl transferase-mediated nick-end labeling) (B) in a prepubertal mouse that received cyclophosphamide $200 \mathrm{mg} / \mathrm{kg} 2$ days earlier. Apoptosis in the granulosa cells surrounding the central oocytes is more evident on TUNEL than on hematoxylin and eosin staining. (C) Mature mouse femur stained with hematoxylin and eosin. (D) Detail of the growth plate section (with computerized measurements). (E) Detail of the growth plate section stained with TUNEL, showing apoptosis.

With permission, from Detti L, Uhlmann RA, Zhang J, et al. Goserelin fosters bone elongation but does not prevent ovarian damage in cyclophosphamide-treated prepubertal mice. Fertil Steril 2014; 101(4): 1157-1164. doi:10.1016/j.fertnstert.2013.12.028

of an antrum, which is an accumulation of fluid. Tertiary follicles are also called antral follicles.

Progression from primordial to tertiary follicles takes approximately 120 days, which is incidentally the same time it takes for the germinal cells that will eventually become sperm to mature.

The stimulus for primordial follicles to enter the first developmental stage and become primary follicles is not well understood. However, an inhibitory hormone, anti-Müllerian hormone (AMH), keeps primordial follicles from entering the first developmental step to become primary follicles. After this first step, primary, secondary, and tertiary follicles are regulated in their development by folliclestimulating hormone (FSH), produced by the pituitary gland. These follicles in turn secrete $\mathrm{AMH}$, which can be considered the gatekeeper of ovarian follicle reserve throughout a female's reproductive life.

\section{OOCYTES ARE LOST OVER TIME, AND WITH CHEMOTHERAPY}

Females are born with all the eggs they will ever have, and the number declines with age. The peak number is actually reached before birth at about 20 weeks of gestation. At that time, the eggs lack the surrounding granulosa cells, and they start organizing into follicles. As this organization progresses, many of the eggs are lost by apoptosis. At birth, there are approximately 2 million follicles, but the number decreases throughout childhood, so that at puberty there are only about 400,000 to 500,000 , and at menopause, basically none.

Any damage to the ovaries-eg, from chemotherapy with alkylating agents such as cyclophosphamide or from radiotherapy-can cause loss of follicles. ${ }^{5}$ Under the microscope, the damage may not be apparent on hematoxylin and eosin staining, but TUNEL staining (terminal nucleotidyl transferase-mediated nick-end labeling) may reveal apoptosis in the nuclei of the granulosa cells (Figure 1), ${ }^{6}$ but not necessarily of the eggs, because it is the granulosa cells that are actively replicating and are therefore more vulnerable to apoptosis than the eggs themselves. ${ }^{7}$

Therefore, systemic chemotherapy tends to directly damage the primary, secondary, and tertiary follicles, which contain more granulosa cells, and these granulosa cells are more metabolically active than those in the primordial follicles, which also contain fewer. However, it also damages primordial follicles through an indirect process. The secondary and tertiary follicles secrete $\mathrm{AMH}$, which inhibits further maturation of primordial follicles. By damaging secondary and tertiary follicles, chemotherapy causes a major decrease in $\mathrm{AMH}$, so that more primordial follicles, lacking this inhibitory signal, enter the next developmental stage, leading to burnout of primordial follicles and depletion of ovarian reserve.

\section{Earlier onset of menopause}

The immediate outcome is fewer follicles, followed by faster loss of follicles as the patient ages than in healthy women. The average age at menopause in the general population is 51 . With high-dose chemotherapy, such as what patients receive in preparation for bone marrow transplant, an 18-year-old patient could lose all 
her follicles at once and go into menopause immediately. A moderate dose could cause an immediate loss of follicles followed by a gradual but still accelerated loss, resulting in menopause before age 51 . With low-dose chemotherapy, the loss is more gradual, but the patient will still go into menopause earlier than normal.

We cannot actually count the follicles in the ovaries of a female patient who undergoes chemotherapy, but we can measure her AMH level to assess ovarian reserve. AMH levels can drop very low soon after the gonadotoxic insult, but over 2 to 3 years they gradually come back up as the primary, secondary, and tertiary follicles start producing it again. After low-dose or moderately low-dose gonadotoxic treatment, AMH levels can return to a plateau and stay there for about 10 to 15 years, but after highly gonadotoxic treatment the plateau is much lower and does not last as long, and eventually these women go into menopause very early. Unfortunately, younger age does not protect against ovarian damage. ${ }^{8}$ Some girls who receive chemotherapy go into menopause before ever reaching puberty.

Of the chemotherapeutic agents, the alkylating agents are considered the most harmful to the gonads, and the damage is dose-dependent. ${ }^{9-11}$ Cancer treatment causes primary ovarian insufficiency, or premature menopause, in about $10 \%$ to $25 \%$ of prepubertal patients and in $36 \%$ of postpubertal patients. ${ }^{12-14}$

With radiotherapy, exposure of the ovaries to about 5 to 20 Grays is enough to cause primary ovarian insufficiency regardless of the age of the patient, and even a dose less than 2 Grays can destroy $50 \%$ of the oocyte reserve, enough to cause infertility if not immediate primary ovarian insufficiency. In addition, uterine irradiation can limit the final adult uterine volume. ${ }^{15}$ This issue should always be addressed when patients undergo radiotherapy to the pelvic area because they could have major complications in pregnancy if they do become pregnant.

\section{PRESERVING FERTILITY}

Young women receiving gonadotoxic therapy have 3 main options for preserving fertility: gonadotropin-releasing hormone (GnRH) analogues, oocyte or embryo cryopreservation, and ovarian tissue cryopreservation.
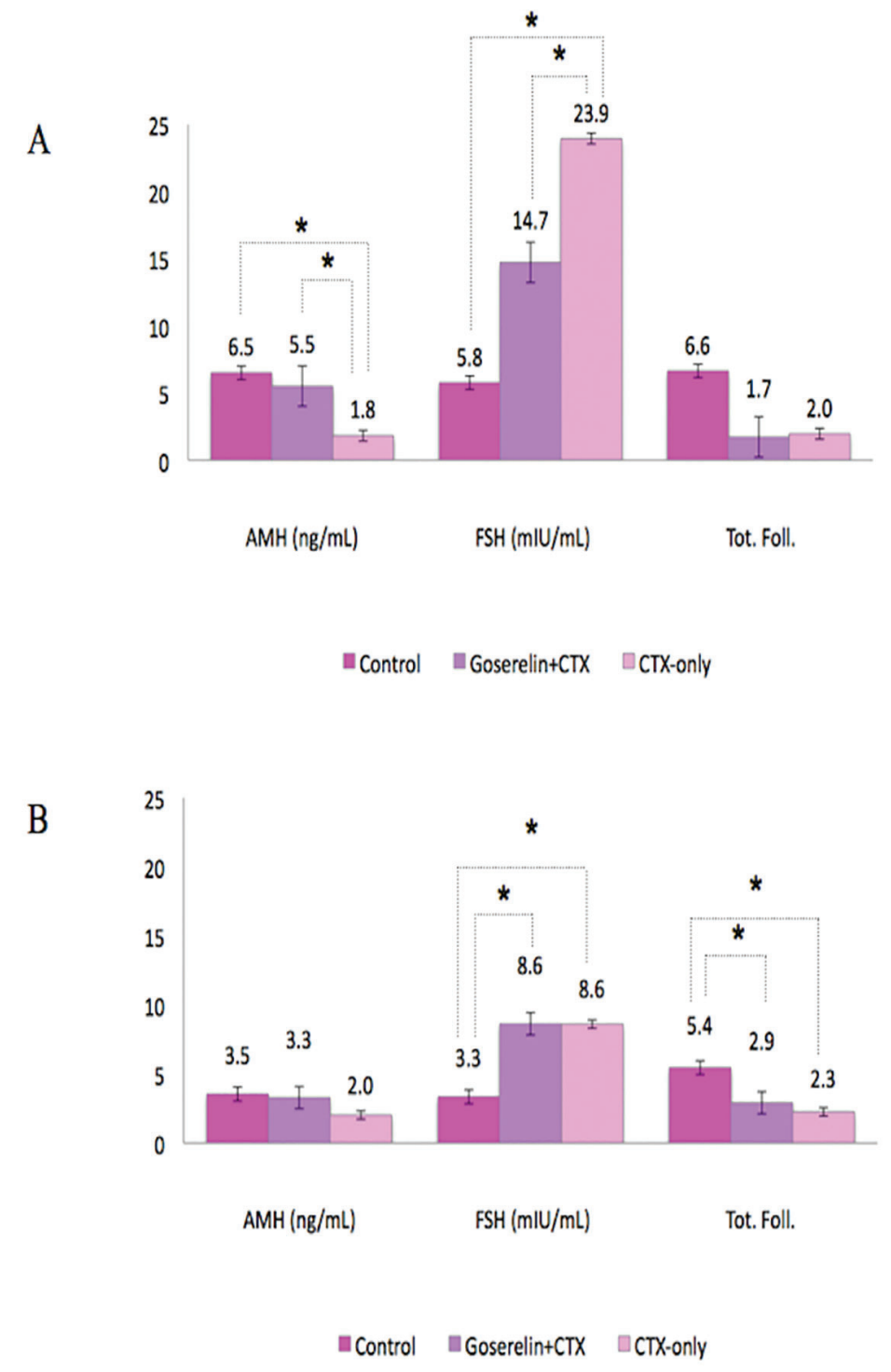

Figure 2. Antimüllerian hormone (AMH) and follicle-stimulating hormone (FSH) levels and total follicle counts at age 56 days $(A)$ and 92 days (B) in mice that had received cyclophosphamide (CTX) alone, CTX plus goserelin, or neither drug (controls) at age 18 days. Goserelin temporarily protected the ovaries, but the protection waned over time $(* P<.05)$.

With permission, from Detti L, Uhlmann RA, Zhang J, et al. Goserelin fosters bone elongation but does not prevent ovarian damage in cyclophosphamide-treated prepubertal mice. Fertil Steril 2014; 101(4): 1157-1164. doi:10.1016/j.fertnstert.2013.12.028

\section{GnRH analogues}

$\mathrm{GnRH}$ analogues have been used for fertility preservation since the 1980s, although their mechanism of action and effectiveness are still debated.

How do they work? Because GnRH ana- 
logues inhibit ovarian function, in theory, they could protect the ovary through several mechanisms:

- Down-regulating ovarian function ${ }^{16}$

- Decreasing utero-ovarian perfusion ${ }^{17,18}$

- Up-regulating intragonadal antiapoptotic factors such as sphingosine 1 phosphate

- Directly protecting developing follicles and germinal cells ${ }^{17-19}$

- Maintaining AMH production, thus keeping primordial follicles in their quiescent state, preventing "burnout," and preserving ovarian reserve. . $^{60}$

In a study of these effects, prepubescent mice (age 20 days) were given either cyclophosphamide alone or cyclophosphamide plus goserelin (a GnRH analogue), while a third group (controls) received neither. ${ }^{6}$ At age 56 days, when the mice were in puberty, $\mathrm{AMH}$ levels were higher in those that received goserelin plus cyclophosphamide than in those who received cyclophosphamide alone, but at 92 days, when the mice were fully mature, AMH levels were similar across the 3 groups (Figure 2). ${ }^{6}$ FSH levels at 56 days were lower with goserelin than with cyclophosphamide alone, though still higher than in the control group, reflecting damage to the ovaries even with goserelin treatment. At 92 days, FSH levels were the same in the 2 groups that got cyclophosphamide and lower than at 56 days, though still higher than in the control group. The total number of follicles was lower in the cyclophosphamide groups with or without goserelin, and lower than in the control group at 92 days. The conclusion was that goserelin did not fully prevent ovarian damage in this mouse model, and that the effect was only temporary, although it did foster bone elongation. ${ }^{6}$

However, in an in vitro experiment, ${ }^{20}$ recombinant $\mathrm{AMH}$ was found to decrease the replication of granulosa cells, leading to the conclusion that if a GnRH analogue decreased damage to the developing follicles, then it could also maintain an AMH level that would prevent that initial burnout of primordial follicles, which would remain a reservoir in the ovaries.

How effective are GnRH analogues? They increase the chances of resuming menses after chemotherapy. However, resumption of menses does not mean fertility, and many times it does not mean that the ovarian reserve is still intact.

A comprehensive review of studies up to $2013^{21}$ found that these agents have a positive impact on resumption of menses. In addition, Lambertini et $\mathrm{al}^{22}$ reviewed randomized clinical trials published up to April 2015 and concluded that luteinizing hormone-releasing factor (the equivalent to a $\mathrm{GnRH}$ analogue) is associated with a significantly reduced risk of primary ovarian failure and "seems to" increase the pregnancy rate. ${ }^{22}$

Limitations of the studies were that they did not all report how long the GnRH analogues were given, the age stratification of the patients, how long after receiving the GnRH analogue the patients became pregnant (there is possibly an immediate effect of GnRH treatment, but perhaps less protection in the long run), or the length of follow-up.

A 2018 American Society of Clinical Oncology guideline ${ }^{23}$ stated that although evidence is conflicting, GnRH agonists may be offered when oocyte or ovarian tissue cryopreservation is not feasible, and in young women with breast cancer. I would go beyond this guideline and offer these agents to girls and women undergoing gonadotoxic therapy for any reason. I agree that $\mathrm{GnRH}$ analogues provide only uncertain or temporary benefits and should be offered only together with other options or if other methods are not feasible. Ideally, they should be given 2 weeks before the start of chemotherapy and should then be held between the chemotherapy cycles to let the ovary recoup follicle development and $\mathrm{AMH}$ production to protect the primordial follicle pool.

\section{Oocyte cryopreservation}

Oocyte cryopreservation (freezing the eggs) is a relatively new technique to preserve fertility in females who have already achieved puberty. It first requires stimulation with gonadotropins for about 10 to 12 days, during which follicular development is followed with transvaginal ultrasonography. Before stimulation, the follicles measure 5 to $10 \mathrm{~mm}$, and when they reach approximately $18 \mathrm{~mm}$, ovulation is induced to allow the eggs to mature.

Only mature eggs at the stage of metaphase 2 can be frozen. Oocytes are retrieved under ultrasonographic guidance, with a long needle 
attached to a transvaginal ultrasound probe. The eggs are frozen (vitrified) and kept in liquid nitrogen, where they can be stored indefinitely. (An ordinary freezer is not cold enough.) When feasible, another option is to fertilize the eggs in vitro immediately after harvesting them and freeze the resulting embryos after they have developed for 5 days, at the blastocyst stage.

When the woman is considered cured of her primary cancer, the eggs or embryos can be thawed and the eggs fertilized in vitro, and then they can be transferred into the uterus under ultrasonographic guidance.

Oocyte cryopreservation was considered experimental until 2012, when the American Society for Reproductive Medicine lifted the experimental label. ${ }^{24}$ Since then, oocyte cryopreservation in female patients undergoing gonadotoxic therapy has become the standard of care.

How many eggs should we obtain and freeze? We don't know the magic number, and the calculation is complicated because the genetic quality of the eggs declines with the age of the patient: ie, the older the woman, the more eggs are needed to allow her to have a pregnancy. Fertility decreases with age even in healthy women, and early pregnancy loss is due in most cases to genetic abnormalities in the oocytes and embryos. At age 22, only about $5 \%$ of eggs have a chromosomal abnormality, but this increases to $22 \%$ at age 32 and $65 \%$ at age 40 to 41 .

Goldman et $\mathrm{al}^{25}$ calculated that at least 10 eggs are needed to provide a $75 \%$ chance of pregnancy at age 34, 20 eggs are needed at age 37 , and 61 are needed at age 42 . Therefore, if we can retrieve about 20 oocytes, at age 34 , there would be about a $90 \%$ chance of pregnancy, decreasing to $75 \%$ at age 37 and to only $37 \%$ at age 42 with oocyte cryopreservation and in vitro fertilization. In comparison, in healthy, sexually active females not using contraception, the probability of becoming pregnant during any given menstrual cycle is only about $20 \%$ to $25 \%$. This technique is thus very effective.

\section{Ovarian tissue cryopreservation}

Ovarian tissue cryopreservation, or freezing the ovary, is a newer method. Usually, the whole ovary is extracted and the ovarian cortex is separated from the medullary portion, which is more vascular. Next, the cortex is sectioned in small fragments and frozen in liquid nitrogen. The ovarian cortex is thawed and transplanted back into the woman after she is cancer-free. ${ }^{26,27}$

An advantage of tissue cryopreservation is that once the ovarian tissue is transplanted back, not only can the patient possibly conceive (just as with egg preservation), but she can also produce her own estrogen and progesterone and will not need hormone replacement therapy to prevent hot flashes, bone loss, and the other consequences of menopause.

Women in primary ovarian insufficiency have very high FSH concentrations, in the menopausal range of $80 \mathrm{mU} / \mathrm{mL}$. After ovarian tissue is transplanted back into the pelvis, the FSH level gradually comes back down to normal levels of 7 to $8 \mathrm{mU} / \mathrm{mL}$ after approximately 6 months, an interval reflecting the 120 days it takes for primordial follicles to develop into tertiary follicles, which are the ones that produce the most estradiol, which feeds back with the FSH produced by the pituitary. ${ }^{28}$

In May 2021, in a report by Dolmans et $\mathrm{al}^{29}$ on the effectiveness of tissue cryopreservation, almost all women who underwent the procedure recovered their ovarian function, and about $25 \%$ gave birth to a healthy child. However, most of them had to undergo in vitro fertilization to become pregnant, and a $25 \%$ pregnancy rate with in vitro fertilization is considered low. Nonetheless, this is a great outcome for patients who underwent cancer therapy. With radiation therapy, the rates of success are much lower than with chemotherapy.

Starting chemotherapy before ovarian tissue cryopreservation does not impair the chances for fertility, and it may reduce the risk of reintroducing cancer with the autotransplant. Especially for blood-borne cancers like leukemia, cancerous cells may lurk in the transplant, but 2 or 3 cycles of chemotherapy before harvesting can lower the risk.

In 2019, the American Society for Reproductive Medicine lifted the experimental label from tissue cryopreservation and now recommends it for clinical practice, particularly for prepubescent patients, for whom oocyte cryopreservation is not possible. ${ }^{30}$ 


\section{OVARIAN TRANSPOSITION}

Ovarian transposition is an established method to protect the ovary from pelvic radiation and to preserve future ovarian function and fertility. The technique, introduced in the 1970s, entails elevation of the ovaries from their pelvic position to an abdominal position. ${ }^{31}$ Today, it can be performed laparoscopi- cally or robotically. However, this procedure should be offered only if the patient is undergoing pelvic radiation as it is not useful if the patient is treated with chemotherapy.

\section{DISCLOSURES}

The author reports no relevant financial relationships which, in the context of her contributions, could be perceived as a potential conflict of interest.

\section{REFERENCES}

1. Magelssen H, Melve KK, Skjaenven R, Fossa SD. Parenthood probability and pregnancy outcome in patients with a cancer diagnosis during adolescence and young adulthood. Hum Reprod 2008; 23(1):178-186. doi:10.1093/humrep/dem362

2. Cvancarova M, Samuelsen SO, Magelssen H, Fossa SD. Reproduction rates after cancer treatment: experience from the Norwegian radium hospital. J Clin Oncol 2009; 27(3):334-343. doi:10.1200/JCO.2007.15.3130

3. Forman EJ, Anders CK, Behera MA. A nationwide survey of oncologists regarding treatment-related infertility and fertility preservation in female cancer patients. Fertil Steril 2010; 94(5):1652-1656. doi:10.1016/j.fertnstert.2009.10.008

4. Hammond C, Abrams JR, Syrjala KL. Fertility and risk factors for elevated infertility concern in 10-year hematopoietic cell transplant survivors and casematched controls. J Clin Oncol 2007; 25(23):3511-3517. doi:10.1200/JCO.2007.10.8993

5. Detti L, Martin DC, Williams RW, Schlabritz-Loutsevich N, Williams $\amalg$ Uhlmann RA. Somatic and reproductive outcomes in mice treated with cyclophosphamide in pre-pubertal age. Syst Biol Reprod Med 2013; 59(3):140-145. doi:10.3109/19396368.2012.751463

6. Detti L, Uhlmann RA, Zhang J, et al. Goserelin fosters bone elongation but does not prevent ovarian damage in cyclophosphamide-treated prepubertal mice. Fertil Steril 2014; 101(4):1157-1164. doi:10.1016/j.fertnstert.2013.12.028

7. Oktem O, Okta K. A novel ovarian xenografting model to characterize the impact of chemotherapy agents on human primordial follicle reserve. Cancer Res 2007; 67(21):10159-10162. doi:10.1158/0008-5472.CAN-07-2042

8. Su HI, Kwan B, Whitcomb BW, et al. Modeling variation in the reproductive lifespan of female adolescent and young adult cancer survivors using AMH. J Clin Endocrinol Metab 2020; 105(8):2740-2751. doi:10.1210/clinem/dgaa172

9. Chemaitilly W, Mertens AC, Mitby P, et al. Acute ovarian failure in the childhood cancer survivor study. J Clin Endocrinol Metab 2006; 91(5):1723-1728. doi:10.1210/jc.2006-0020

10. Green DM, Sklar CA, Boice JD Jr, et al. Ovarian failure and reproductive outcomes after childhood cancer treatment: results from the Childhood Cancer Survivor Study. J Clin Oncol 2009; 27(14):2374-2381. doi:10.1200/JCO.2008.21.1839

11. Hudson MM. Reproductive outcomes for survivors of childhood cancer. Obstet Gynecol 2010; 116(5):1171-1183. doi:10.1097/AOG.0b013e3181f87c4b

12. Revel A, Revel-Vilk S, Aizenman E, et al. At what age can human oocytes be obtained? Fertil Steril 2009; 92(2):458-463. doi:10.1016/j.fertnstert.2008.07.013

13. Jadoul P, Dolmans M-M, Donnez J. Fertility preservation in girls during childhood: is it feasible, efficient and safe and to whom should it be proposed? Hum Reprod Update 2010; 16(6):617-630. doi:10.1093/humupd/dmq010

14. Hudson MM, Ness KK, Gurney JG, et al. Clinical ascertainment of health outcomes among adults treated for childhood cancer. JAMA 2013; 309(22):23712381. doi:10.1001/jama.2013.6296

15. Critchley HOD, Wallace WHB. Impact of cancer treatment on uterine function. J Natl Cancer Inst Monogr 2005; 34:64-68. doi:10.1093/jncimonographs/lgi022

16. Blumenfeld Z, Avivi I, Ritter M, Rowe JM. Preservation of fertility and ovarian function and minimizing chemotherapy-induced gonadotoxicity in young women. J Soc Gynecol Invest 1999; 6(5):229-239. doi:10.1016/s1071-5576(99)00028-3

17. Blumenfeld $Z$. How to preserve fertility in young women exposed to chemotherapy? The role of $\mathrm{GnRH}$ agonist cotreatment in addition to cryopreservation of embrya, oocytes, or ovaries. Oncologist 2007; 12(9):1044-1054. doi:10.1634/theoncologist.12-9-1044

18. Meirow D, Assad G, Dor J, Rabinovici J. The GnRH antagonist cetrorelix reduces cyclophosphamide-induced ovarian follicular destruction in mice. Hum Reprod 2004; 19(6):1294-1299. doi:10.1093/humrep/deh257

19. Blumenfeld Z. Fertility preservation using $\mathrm{GnRH}$ agonists: rationale, possible mechanisms, and explanation of controversy. Clin Med Insights Reprod Health 2019; 13:1179558119870163. doi:10.1177/1179558119870163

20. Detti L, Abuzeid MI, Peregrin-Alvarez I, et al. Recombinant anti-Müllerian hormone (rAMH) for stalling in vitro granulosa cell replication. Reprod Sci 2020; 10:1873-1878. doi:10.1007/s43032-020-00206-6

21. Osborne SE, Detti L. GnRH-analogues for ovarian protection in childhood cancer patients: how adult hypotheses are relevant in prepubertal females. Curr Drug Targets 2013; 14(8):856-863. doi:10.2174/1389450111314080005

22. Lambertini M, Ceppi M, Poggio F, et al. Ovarian suppression using luteinizing hormone-releasing hormone agonists during chemotherapy to preserve ovarian function and fertility of breast cancer patients: a meta-analysis of randomized studies. Ann Oncol 2015; 26(12):2408-2419. doi:10.1093/annond/mdv374

23. Oktay K, Harvey BE, Partridge AH, et al. Fertility preservation in patients with cancer: ASCO clinical practice guideline update. J Clin Oncol 2018; 36(19):1994-2001. doi:10.1200/JCO.2018.78.1914

24. Practice Committees of the American Society for Reproductive Medicine and the Society for Assisted Reproductive Technology. Mature oocyte cryopreservation: a guideline. Fertil Steril 2013; 99(1):37-43. doi:10.1016/j.fertnstert.2012.09.028

25. Goldman RH, Racowsky C, Farland LV, Munne S, Ribustello L, Fox JH. Predicting the likelihood of live birth for elective oocyte cryopreservation: a counseling tool for physicians and patients. Hum Reprod 2017; 32(4):853-859. doi:10.1093/humrep/dex008

26. Donnez J, Dolmans M-M. Cryopreservation and transplantation of ovarian tissue. Clin Obstet Gynecol 2010; 53(4):787-796. doi:10.1097/GRF.0b013e3181f97a55

27. Silber S, Kagawa N, Kuwayama M, Gosden R. Duration of fertility after fresh and frozen ovary transplantation. Fertil Steril 2010; 94(6):2191-2196. doi:10.1016/j.fertnstert.2009.12.073

28. Donnez J, Silber S, Andersen CY, et al. Children born after autotransplantation of cryopreserved ovarian tissue. A review of 13 live births. Ann Med 2011; 43(6):437-450. doi:10.3109/07853890.2010.546807

29. Dolmans M-M, von Wolff M, Poirot C, et al. Transplantation of cryopreserved ovarian tissue in a series of 285 women: a review of five leading European centers. Fertil Steril 2021; 115(5):1102-1115. doi:10.1016/j.fertnstert.2021.03.008

30. Practice Committee of the American Society for Reproductive Medicine. Fertility preservation in patients undergoing gonadotoxic therapy or gonadectomy: a committee opinion. Fertil Steril 2019; 112(6):1022-1033. doi:10.1016/j.fertnstert.2019.09.013

31. Nahhas WA, Nisce LZ, D’Angio GJ, Lewis JL Jr. Lateral ovarian transposition. Ovarian relocation in patients with Hodgkin's disease. Obstet Gynecol 1971; 38(5):785-788. pmid:5114230

ADDRESS: Laura Detti, MD, Department of Subspecialty Care for Women's Health A81, Cleveland Clinic, 9500 Euclid Avenue, Cleveland, OH 44195; dettil@ccf.org 